\title{
Pipeline embolization device (PED) for neurovascular reconstruction: initial experience in the treatment of 101 intracranial aneurysms and dissections
}

\author{
Sebastian Fischer • Zsolt Vajda • Marta Aguilar Perez • \\ Elisabeth Schmid • Nikolai Hopf • Hansjörg Bäzner • \\ Hans Henkes
}

Received: 15 February 2011 / Accepted: 27 July 2011 /Published online: 1 September 2011

(C) The Author(s) 2011. This article is published with open access at Springerlink.com

\begin{abstract}
Introduction The purpose of this study was to evaluate the safety and efficacy of the recently available flow diverter "pipeline embolization device" (PED) for the treatment of intracranial aneurysms and dissections.

Methods Eighty-eight consecutive patients underwent an endovascular treatment of 101 intracranial aneurysms or dissections using the PED between September 2009 and January 2011. The targeted vessels include 79 (78\%) in the anterior circulation and $22(22 \%)$ in the posterior circulation. We treated 96 aneurysms and 5 vessel dissections. Multiple devices were implanted in 67 lesions (66\%).

Results One technical failure of the procedure was encountered. Immediate exclusion of the target lesion was not observed. Angiographic follow-up examinations were carried out in 80 patients $(91 \%)$ with 90 lesions and revealed complete cure of the target lesion(s) in 47 (52\%), morphological improvement in 32 lesions (36\%), and no improvement in 11 lesions (12\%). Six major complications were encountered: one fatal aneurysm rupture, one acute and one delayed PED thrombosis, and three hemorrhages in the dependent brain parenchyma.

Conclusion Our experience reveals that the PED procedure is technically straightforward for the treatment of
\end{abstract}

S. Fischer $\cdot$ Z. Vajda $\cdot$ M. Aguilar Perez $\cdot$ H. Henkes $(\bowtie)$

Neuroradiologische Klinik, Neurozentrum, Klinikum Stuttgart,

Stuttgart, Germany

e-mail: hhhenkes@aol.com

E. Schmid $\cdot$ H. Bäzner

Neurologische Klinik, Neurozentrum, Klinikum Stuttgart,

Stuttgart, Germany

N. Hopf

Neurochirurgische Klinik, Neurozentrum, Klinikum Stuttgart,

Stuttgart, Germany selected wide-necked saccular aneurysms, fusiform aneurysms, remnants of aneurysms, aneurysms with a high likelihood of failure with conventional endovascular techniques, and dissected vessels. While vessel reconstruction, performed after dissection, is achieved within days, remodeling of aneurysmal dilatations may take several months. Dual platelet inhibition is obligatory. Parenchymal bleeding into brain areas dependent on the target vessel is uncommon.

Keywords Flow diverter Pipeline embolization device. Aneurysm · Dissection

\section{Introduction}

Endovascular treatment has matured to a safe and effective therapy in the management of intracranial aneurysms. In saccular aneurysms with a well-defined neck, coil occlusion can mostly be achieved without difficulty. In wide-necked aneurysms, stent deployment or balloon remodeling may be required in order to exclude the aneurysm with preservation of the parent artery $[1,2]$.

The term "flow diversion" refers to the concept of hemodynamic influence on an aneurysm by reducing both inflow and outflow. Flow modification and reconstruction of the parent artery with subsequent intraaneurysmal thrombosis or "reverse remodeling" can, within certain limits, be achieved by conventional porous stents and stent grafts [3-5]. The main issues of stent grafts are their stiffness and the unavoidable occlusion of all covered side branches. The technique of telescoping porous stents may work, but efficiency mostly remains unpredictable [6].

Flow diverters are stent-like endovascular implants designed to alter the blood flow in an artery in a way that 
favors the flow along the longitudinal axis of the target vessel and reduces inflow and outflow of an aneurysm covered by the device. Flow diverters can be based on braided or laser-cut metallic structures. The first flow diverter commercially available was the Silk (Balt Extrusion, Montmorency, France) [7]. A tight mesh of small cell sizes and a high longitudinal flexibility offer the possibility to cover an aneurysm orifice. In selected cases, flow diverters may also be used successfully to reconstruct intracranial vessel dissections.

In 2010, two different flow diverter devices met the requirements of the Medical Devices Directive and were available with CE mark (Silk, Balt Extrusion, Montmorency, France; Pipeline Embolization Device, PED, ev3, Irvine, CA, USA). In this study, we sought to evaluate our initial experience with the PED in a series of 88 patients with 101 treated intracranial vascular lesions.

\section{Material and methods}

\section{Pipeline embolization device}

The PED used in this series is a composite braided mesh tube of 48 strands with $75 \%$ cobalt chromium and $25 \%$ platinum. The single wire has a diameter of $30 \mu \mathrm{m}$. Devices are available with a nominal diameter from 2.5 to $5 \mathrm{~mm}$ with $0.25 \mathrm{~mm}$ increments. The nominal length of the implants is in the range from 10 to $35 \mathrm{~mm}$ in $2 \mathrm{~mm}$ increments for the 10 - to 20 - $\mathrm{mm}$ lengths and in $5 \mathrm{~mm}$ increments for the 25 - to $35-\mathrm{mm}$ lengths. At the nominal diameter, the pore size is 0.02 $0.05 \mathrm{~mm}^{2}$ and the radial force is about $2.0 \mathrm{mN} / \mathrm{mm}(3.0 \mathrm{~mm}$ vessel diameter), which is similar to a Silk stent. The braided wires are loose on both ends. The PED is premounted on a stainless steel wire and is attached distally to a capture coil. A radiopaque $15-\mathrm{mm}$ platinum tip extends beyond the end of the PED. Both the guide wire and the PED are inserted via a 0.027-in. ID microcatheter (Marksman, ev3, Irvine, CA, USA). Once the PED has reached the desired position, deployment starts by gently withdrawing the Marksman catheter and simultaneously advancing the insertion wire. Expansion of the PED results in gradual foreshortening which pulls the distal end of the device out of the capture coil. As soon as the distal end of the PED is detached from the capture coil, further deployment is a combination of advancing the insertion wire and passive backward migration of the microcatheter. Since the density of vessel wall coverage is largely dependent on the sizing of the implant, accurate vessel diameter measurement is mandatory. Oversizing the device will result in decreased wall coverage and reduced hemodynamic effect. Based on previous reports and on our own experience, several PEDs may be required to achieve a significant hemodynamic effect.
Selection criteria and patient population

A total of 88 patients with 101 intracranial lesions were treated with the PED between September 2009 and January 2011 in a single neurovascular center. During this period, 94 aneurysms were treated microsurgically in our institution. Ninety-six aneurysms were treated with PED, and a total of 195 conventional (i.e., coils and/or stents) endovascular aneurysm procedures were performed. Treatment decision was based on clinical and anatomical considerations and on interdisciplinary discussion between the neurovascular team members. Only unruptured aneurysms or remnants of previously treated aneurysms were considered for PED treatment due to the medical platelet antiaggregation therapy required. Indications for treatment with the PED were wide neck, aneurysms with a high likelihood of failure with conventional endovascular or surgical techniques, remnants of aneurysms after surgical or endovascular treatment, fusiform aneurysms, and dissected vessels.

Patients were not selected according to age or other demographic factors. An absolute exclusion criterion for PED treatment was acute aneurysm rupture. Relative exclusion criteria were anticipated ease of either microsurgical or conventional endovascular treatment, severe elongation of the supraaortic vessels, and confirmed intolerance or resistance to clopidogrel and/or acetylsalicylic acid (ASA).

\section{Clinical presentation and lesion morphology}

Of the 101 treated lesions, $61(60 \%)$ were found incidentally, $18(18 \%)$ were detected due to a previous subarachnoid hemorrhage (SAH) from the treated or another aneurysm, $9(9 \%)$ presented with cranial nerve palsy, and $13(13 \%)$ were symptomatic dissections that had caused either cerebral ischemia or $\mathrm{SAH}$.

The series comprises 63 (62\%) saccular aneurysms, 33 (33\%) fusiform aneurysms, and $5(5 \%)$ dissections. The mean fundus diameter of the 63 saccular aneurysms was $3.8 \mathrm{~mm}$ (range, 2.0 to $15.0 \mathrm{~mm}$; standard deviation, $2.9 \mathrm{~mm}$ ) and the mean diameter of the aneurysm neck was $3.3 \mathrm{~mm}$ (range, 2.0 to $15.0 \mathrm{~mm}$; standard deviation, $2.4 \mathrm{~mm}$ ). Of the 63 saccular aneurysms treated in this series, the largest fundus diameter was $<5 \mathrm{~mm}$ in $n=49,5-10 \mathrm{~mm}$ in $n=11$, and $>10 \mathrm{~mm}$ in $n=3$ aneurysms. The mean diameter of the parent artery proximal to the aneurysm was $4.0 \mathrm{~mm}$ (range, $2.0-7.0 \mathrm{~mm}$; standard deviation, $1.0 \mathrm{~mm}$ ) and the parent artery distal from the aneurysm had a mean diameter of $3.3 \mathrm{~mm}$ (range, 2.0 $5.0 \mathrm{~mm}$; standard deviation, $0.7 \mathrm{~mm}$ ). In patients with a diameter $>5 \mathrm{~mm}$ of the target vessel adjacent to the target lesion, the "landing zone" of the proximal end of the PED was intentionally extended more proximally in order to provide dense wall apposition and avoid an "endoleak". 
A total of $79(78 \%)$ treated lesions were located in the anterior circulation and $22(22 \%)$ were located in the posterior circulation. In the anterior circulation, 6 out of 74 aneurysms (saccular and fusiform aneurysms) were located outside the dura.

\section{Previous treatment}

All previous treatments and/or underlying dissections were recorded in a patient database.

\section{Clinical presentation and follow-up}

The clinical course of each patient was evaluated according to the modified Rankin Scale (mRS) before treatment, at discharge, and at each follow-up angiography (scheduled for 3 and 6 months after the initial treatment).

\section{Endovascular procedure}

The primary intention of every procedure was complete coverage across the entire length of the dilated or dissected vessel segment with the PED(s). Procedures were performed on a biplane flat panel digital subtraction angiography (DSA) unit (Allura Xper 20/10, Philips, Einthoven, The Netherlands; Axiom Artis, Siemens, Erlangen, Germany).

All procedures were carried out under general anesthesia. Patients received a loading dose of $500 \mathrm{mg}$ ASA and $600 \mathrm{mg}$ clopidogrel at least 1 day prior to the treatment and a dual antiplatelet treatment with $100 \mathrm{mg}$ ASA and $75 \mathrm{mg}$ clopidogrel daily thereafter. Each patient was tested for proper response to ASA and clopidogrel with either Verify Now $^{\circledR}$ or Multiplate ${ }^{\circledR}$. Only one patient was found to be nonresponder to clopidogrel and was given prasugrel instead. The patient tolerated the procedure well despite the need to treat with five PEDs. An intravenous bolus of heparin $(5,000 \mathrm{IU})$ and $500 \mathrm{mg}$ ASA were given at the beginning of the procedure. Activated clotting time was not routinely measured. All procedures were carried out or supervised by the senior author.

The parent artery was catheterized with a 6-F guide catheter or with a telescoping system of $8 \mathrm{~F} / 6 \mathrm{~F}$ distal access guide catheters. A Marksman microcatheter (ev3, Irvine, CA, USA) was inserted into the parent artery distally to the aneurysm with a 0.014 - or 0.016 -in. microguidewire. The distal end of the microcatheter was positioned in a straight segment of the parent artery distal to the target site in order to avoid any vessel injury by the distal end of the PED delivery system tip wire.

The PED was advanced through the microcatheter and positioned at the level of the aneurysm. To deploy the device, the microcatheter was pulled back along with simultaneous advancement of the insertion wire to keep the PED in place. The correct expansion of the PED was documented under fluoroscopy and, in most of the cases, with additional angiographic computed tomography (DynaCT).

Postprocedural medication included permanent medication with $100 \mathrm{mg}$ ASA daily. At the beginning of this series, $75 \mathrm{mg}$ clopidogrel daily were administered for 6 weeks. After delayed thromboembolic events following flow diverter treatment had been reported [8], we increased the duration of clopidogrel medication in March 2010 for at least 1 year. All patients that had been treated before March 2010 were informed accordingly. The treatment results in saccular aneurysms were evaluated according to the O’Kelly-Marotta (OKM) grading scale [9].

\section{Results}

The series includes 45 female and 43 male patients with a mean age of 59 years. Treatment with the PED was performed in 54 (53\%) previously untreated lesions. There were $30(30 \%)$ lesions that had been previously treated with laser-cut porous stents, $9(9 \%)$ remnants that had been previously coiled, and $8(8 \%)$ remnants that had been previously clipped. Intracranial dissections were treated with the PED in five patients.

All lesions were successfully accessed in this series, and successful deployment of the devices was achieved in all but one procedure (technical success rate, 99\%). The failed attempt was a retreatment of a wide-necked aneurysm of the internal carotid artery (ICA) located at the origin of the posterior communicating artery. The PED could not be placed distally enough due to a major elongation of the supraaortic vasculature. Since the PED did not cover the aneurysm completely, the aneurysm was subsequently covered with an Enterprise stent (Codman, Raynham, MA, USA).

Balloon dilatation of the target vessel prior to the PED deployment was performed in eight patients with preexisting stenosis (Fig. 1). Balloon dilatation after incomplete expansion of the device(s) was carried out in 13 cases. Partial expansion of the PED was mainly observed in vessel segments with tight or acute curves (Fig. 2).

More than one PED was implanted (range, 2-18; median, 3.0) in 67 out of 101 procedures (66\%). The decision to implant more than one device was based on anatomical circumstances or on the angiographic aspect after implantation of one or several PEDs (Fig. 3). Indications for the placement of multiple devices were (1) unchanged aneurysm perfusion, (2) a deceleration of contrast circulation within the aneurysm without contrast stagnation into the venous phase, or (3) specially in cases of fusiform aneurysms, an incomplete reconstruction of the 

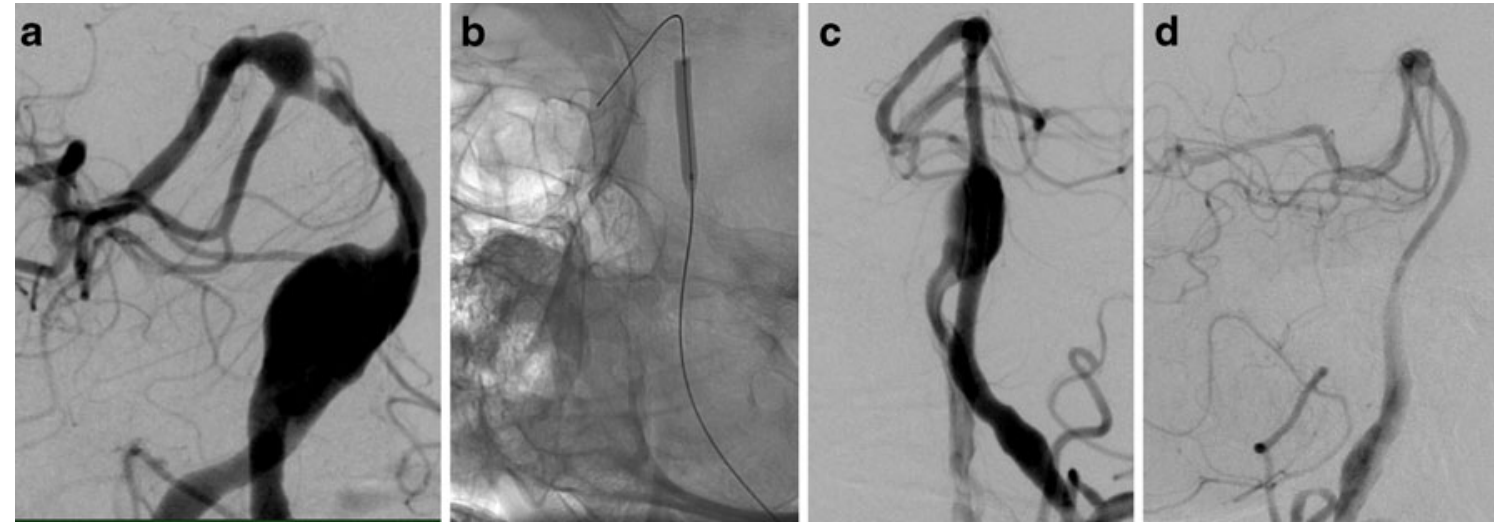

Fig. 1 Fusiform dilatation of the basilar artery with associated stenosis (a). Preparatory balloon angioplasty (b), followed by PED deployment $[3 \times 4.5 \times 20,4.75 \times 20,4.75 \times 18,5.0 \times 20,3.5 \times 20$,

parent artery. According to this strategy, we limited the procedure to a single device if reduced aneurysm filling or contrast stagnation was visible until the venous phase, insinuating a high likelihood of intraaneurysmal thrombo-
$3.0 \times 20]$ (c). Angiographic follow-up 4 months later shows remodeling of the dilated vessel (d)

sis. Complete aneurysm occlusion immediately after PED deployment was not observed in any of the patients.

Additional coiling in the same session was performed in three cases with a microcatheter that was placed within the
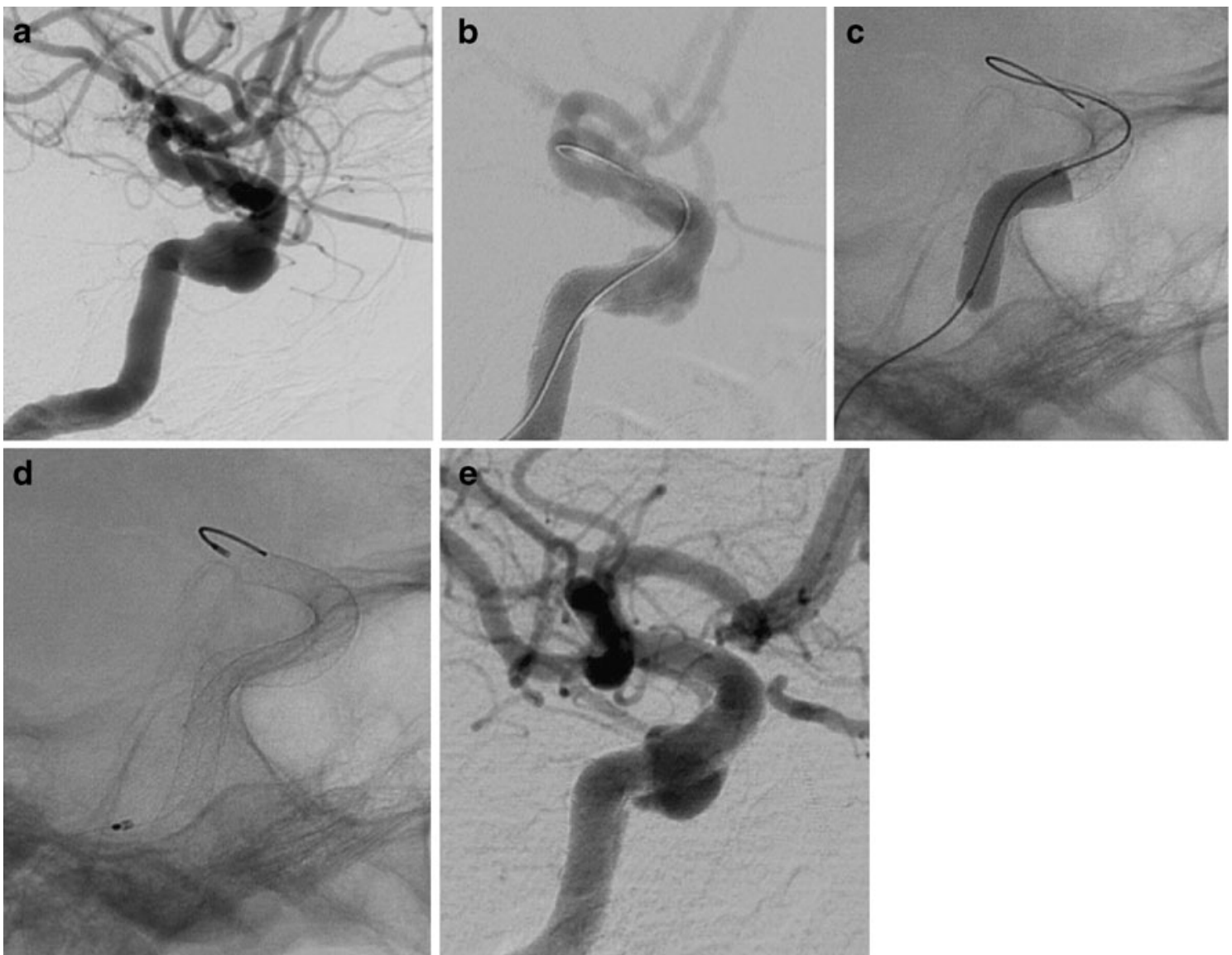

Fig. 2 Cavernous aneurysm of the right ICA (a). Poor wall apposition after deployment of the second PED [2 $\times 4.5 / 20]$ (b). Adaptation of the PEDs to the vessel wall using a $4 \times 15$-mm Ascent balloon (c) results in secondary expansion of the second device with close contact between its outer surface and the vessel wall (d). Angiographic follow-up 3 months later shows a decrease of the aneurysm size and a regular reconstruction of the parent artery (e) 

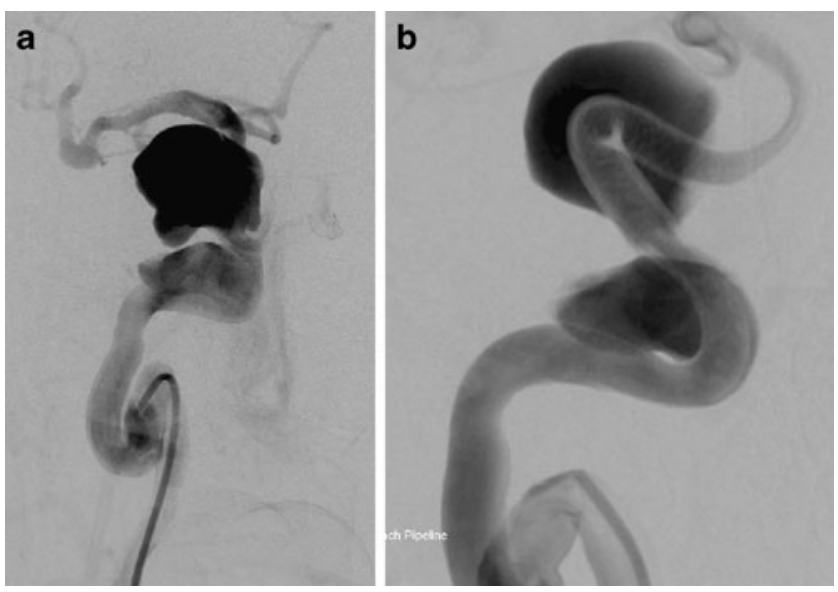

Fig. 3 Deployment of 19 PEDs in a patient with large aneurysms of the right ICA, presenting with diplopia and severe headache. Injection of the right ICA after direct puncture shows fusiform aneurysms of the petrous and cavernous segments separated by a relative stenosis (a). Reconstruction of the vessel with 19 PEDs after preparatory balloon angioplasty (b). MRI 3 days later shows thrombosis of both aneurysms. In the meantime, the patient has improved clinically

aneurysm before implantation of the PED ("jailed catheter"). In these cases, additional coiling was performed since the effect of flow diversion without additional coiling was thought to be insufficient. We did not observe any clinically relevant side branch occlusions on post-interventional or follow-up angiograms among all treated lesions.

\section{Follow-up}

Each patient was scheduled for follow-up angiography 3 and 6 months after treatment. The clinical condition was evaluated according to the mRS. At least 1 DSA follow-up was available in $80(91 \%)$ patients with $90(89 \%)$ lesions. The mean followup time until the first DSA was 3 months (range, 1-7 months).

The angiographic follow-up results were classified according to the OKM grading scale [9]. This scale accounts for the amount of aneurysm perfusion and stasis of contrast medium. Aneurysm perfusion is graded as: A, complete perfusion (>95\%); B, incomplete perfusion (5-95\%); C, neck remnant $(>5 \%)$; or $\mathrm{D}$, no perfusion $(0 \%)$. The contrast medium stagnation grade is determined by the timing of contrast medium clearance from the aneurysm sac as defined by the phases of the angiogram: 1, no stasis (clearance within the arterial phase); 2, moderate stasis (clearance prior to the venous phase); 3 , significant stasis (contrast persists in the aneurysm into the venous phase and beyond).

Angiographic results according to the OKM grading scale at the first and last follow-up DSA are shown in Table 1. Two patients with an occlusion of the target vessel and one patient with an aneurysm rupture cannot be classified. There was one de novo aneurysm formation observed after the treatment of a dissection.

More than one follow-up DSA was carried out in 41 (47\%) patients and in $49(49 \%)$ lesions. The mean follow-up time to the last DSA was 10 months (range, 1-15 months). There were three patients with mild, clinically asymptomatic intimal hyperplasia observed on follow-up angiography.

After 3 months, 90 out of 101 lesions had undergone follow-up DSA and 47 (52\%) out of these 90 lesions were angiographically occluded. After an average of 10 months, 49 lesions underwent at least a second DSA and 36 of these 49 lesions (74\%) were angiographically obliterated, including 25 lesions in which the first DSA had already shown occlusion. Eleven of the 36 (31\%) lesions were perfused on the first follow-up DSA and improved to complete occlusion until the 10-month follow-up DSA (Fig. 4). There was no recurrent perfusion once complete obliteration had been achieved.

Adverse events during the procedure and/or during the follow-up occurred in 1 out of 54 treatments $(2 \%)$ without a previous stent placement and in 4 out of 30 treatments (13\%) in whom a PED was deployed inside another stent. The observed occlusion rate was $69 \%$ and $65 \%$ for aneurysms without and with another stent, respectively.
Table 1 Angiographic follow-up results after PED treatment: volume of contrast filling of the aneurysm and degree of stasis of contrast material at the $\{$ first $\}$ and [last] follow-up DSA according to the OKM grading scale

\begin{tabular}{llll}
\hline Volume of contrast filling & \multicolumn{2}{l}{ Degree of stasis } & \\
\cline { 2 - 4 } & 1, no stasis & 2, moderate stasis & 3, significant stasis \\
\hline A, complete & $\{n=8(9 \%)\}$ & $\{n=1(1 \%)\}$ & $\{n=2(2 \%)\}$ \\
B, subtotal & {$[N=1(2 \%)]$} & {$[N=0(0 \%)]$} & {$[N=1(2 \%)]$} \\
& $\{n=9(10 \%)\}$ & $\{n=4(5 \%)\}$ & $\{n=8(9 \%)\}$ \\
C, entry remnant & {$[N=3(6 \%)]$} & {$[N=1(2 \%)]$} & $\{n=1(1 \%)\}$ \\
& $\{n=4(5 \%)\}$ & $\{n=3(3 \%)\}$ & {$[N=0(0 \%)]$} \\
D, no filling & {$[N=3(6 \%)]$} & {$[N=1(2 \%)]$} & \\
Occlusion of target vessel & $\{n=47(52 \%)\}$ & & \\
Aneurysm rupture & {$[N=36(74 \%)]$} & & \\
\hline
\end{tabular}



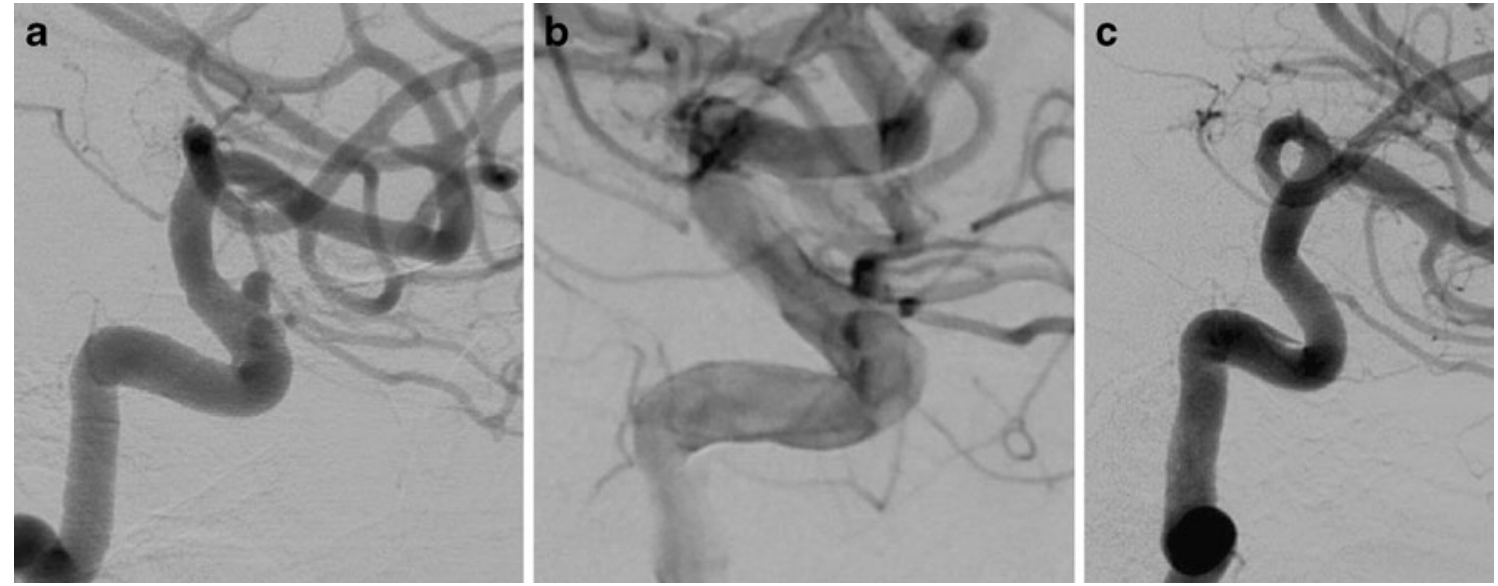

Fig. 4 Progressive vessel remodeling after flow diversion. Injection of the left ICA with a paraophthalmic aneurysm (a). Deployment of three PEDs induced contrast stagnation in the aneurysm (b). After 4 months, complete obliteration of the aneurysm was confirmed (c)

\section{Clinical outcome}

Table 2 summarizes the clinical pre- and post-interventional status in a 3-month period according to the mRS. Clinical impairment prior to discharge occurred in six patients $(6 \%)$ and was due to:

- bleeding from the treated aneurysm (one patient),

- thrombo-embolic events (two patients),

- hemorrhage in the vasculature distal to the target vessel (two patients),

- gastrointestinal bleeding after the procedure (one patient).

Technical complications without clinical sequels were encountered in three procedures. The rates of procedurerelated morbidity and mortality in the entire series to date (January 2011) are 4\% (four patients) and 2\% (two patients), respectively. The procedural complication rate and the occlusion rate during follow-up for saccular aneurysms with a fundus diameter $<5 \mathrm{~mm}$ was $2 \%$ and $86 \%$, respectively.

\section{Retreatment}

A second PED treatment was carried out in eight patients $(9 \%)$. The reason for a second treatment session in all patients was the persistent or unchanged aneurysm perfusion on follow-up angiography and one de novo aneurysm formation after the treatment of a dissection of the ICA.

\section{Illustrative cases}

Patients are numbered according to the date of their first treatment. These numbers will be used here and elsewhere.

Patient 5 This 74-year-old female patient presented with symptoms of brainstem compression due to a large fusiform aneurysm of the basilar trunk. Immediately after the deployment of two PEDs $(3 \times 18 \mathrm{~mm})$, contrast stagnation was observed within the aneurysm. The follow-up DSA performed 8 months after the treatment demonstrated an incomplete occlusion of the aneurysm, with a wellreconstructed lumen of the basilar artery and patent anterior inferior cerebellar arteries (AICAs). At 13 months, reperfu-
Table 2 Clinical status before and after PED treatment

\begin{tabular}{lcccc}
\hline mRS & Pretreatment $(N=88)$ & At discharge $(n=88)$ & 90 days $(n=80)$ & Last follow-up $(n=41)$ \\
\hline 0 & $64(73 \%)$ & $63(71 \%)$ & $63(79 \%)$ & $36(88 \%)$ \\
1 & $14(16 \%)$ & $11(13 \%)$ & $7(9 \%)$ & $2(6 \%)$ \\
2 & $8(9 \%)$ & $7(8 \%)$ & $2(3 \%)$ & $0(0 \%)$ \\
3 & $0(0 \%)$ & $0(0 \%)$ & $1(1 \%)$ & $1(2 \%)$ \\
4 & $2(2 \%)$ & $4(5 \%)$ & $5(6 \%)$ & $1(2 \%)$ \\
5 & $0(0 \%)$ & $1(1 \%)$ & $1(1 \%)$ & $0(0 \%)$ \\
6 & $0(0 \%)$ & $2(2 \%)$ & $1(1 \%)$ & $1(2 \%)$ \\
\hline
\end{tabular}



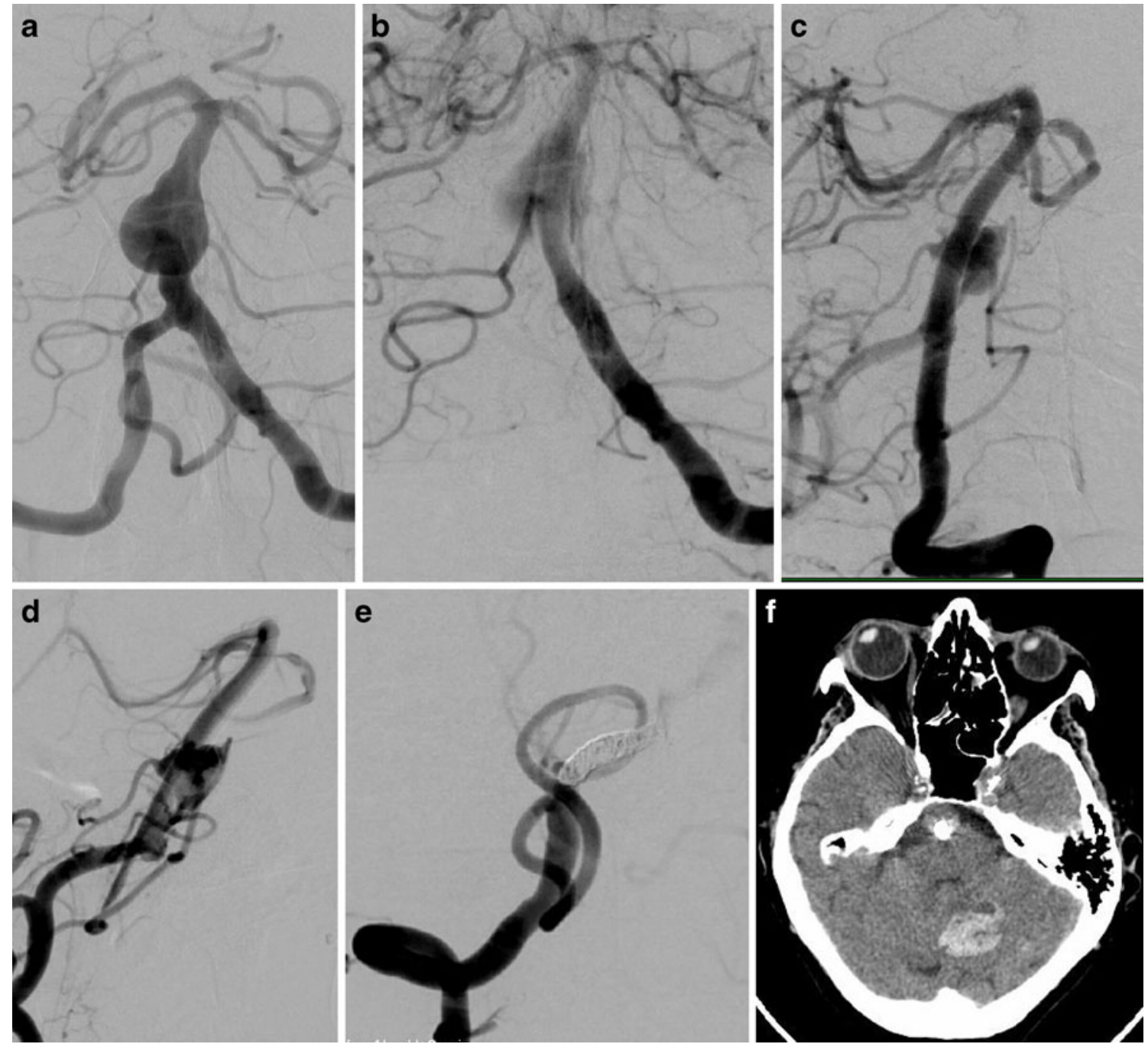

Fig. 5 Large fusiform aneurysm of the basilar trunk with brainstem compression (a). Contrast stagnation within the aneurysm after PED deployment [2 $\times 3 / 18 \mathrm{~mm}$ ] (b). Shrinkage of the aneurysm 8 months later with preserved AICAs (c). Reperfusion and recurrent growth of

sion of the aneurysm was found, which was related to the flow coming from the right vertebral artery (VA), creating turbulent flow around the outer surface of the PEDs. The right V4 segment was, therefore, occluded with coils. On day 1 post treatment, the patient suffered a severe cerebellar hemorrhage, from which she recovered completely (Fig. 5).

Patient 33 This 56-year-old female patient with a history of SAH had previously been treated surgically for several aneurysms, including clipping of a paraclinoid aneurysm of the right ICA. Angiographic follow-up showed, among others, four wide-necked aneurysms in the paraclinoid and supraclinoid segment of the elongated right ICA. Surgical retreatment and coil occlusion were considered difficult due to the previous operation, vessel elongation and aneurysm geometry. After the coaxial the fusiform aneurysm 11 months after the treatment $(\mathbf{d})$ induced by flow coming from the right VA. Coil occlusion of the right V4 segment (e). Cerebellar hemorrhage 1 day after coil occlusion treated conservatively (f)

deployment of two PEDs $(2.5 \times 20 \mathrm{~mm})$, DSA showed stasis of contrast material in the aneurysms. DynaCT confirmed the full expansion of the PEDs. Complete exclusion of the four aneurysms with reconstruction of the lumen of the right ICA was confirmed at the first follow-up DSA after 4 months (Fig. 6).

\section{Complications}

Severe procedural complications were encountered in six patients:

Patient 2: massive intraventricular hemorrhage (IVH) on the third day after combined PED and coil treatment of a wide-necked paraclinoid aneurysm 
Fig. 6 Four de novo aneurysms of the right paraclinoid and supraclinoid ICA, following microsurgical clipping of an aneurysm in the same location years ago (a). Stasis of contrast material in the aneurysms after deployment of two PEDs $[2 \times 2.5 /$ $20 \mathrm{~mm}$ ] (b). DynaCT confirmed the full expansion of the PEDs (c). Obliteration of the four aneurysms with reconstruction of the lumen of the right ICA was confirmed at the first follow-up DSA after 4 months (d)
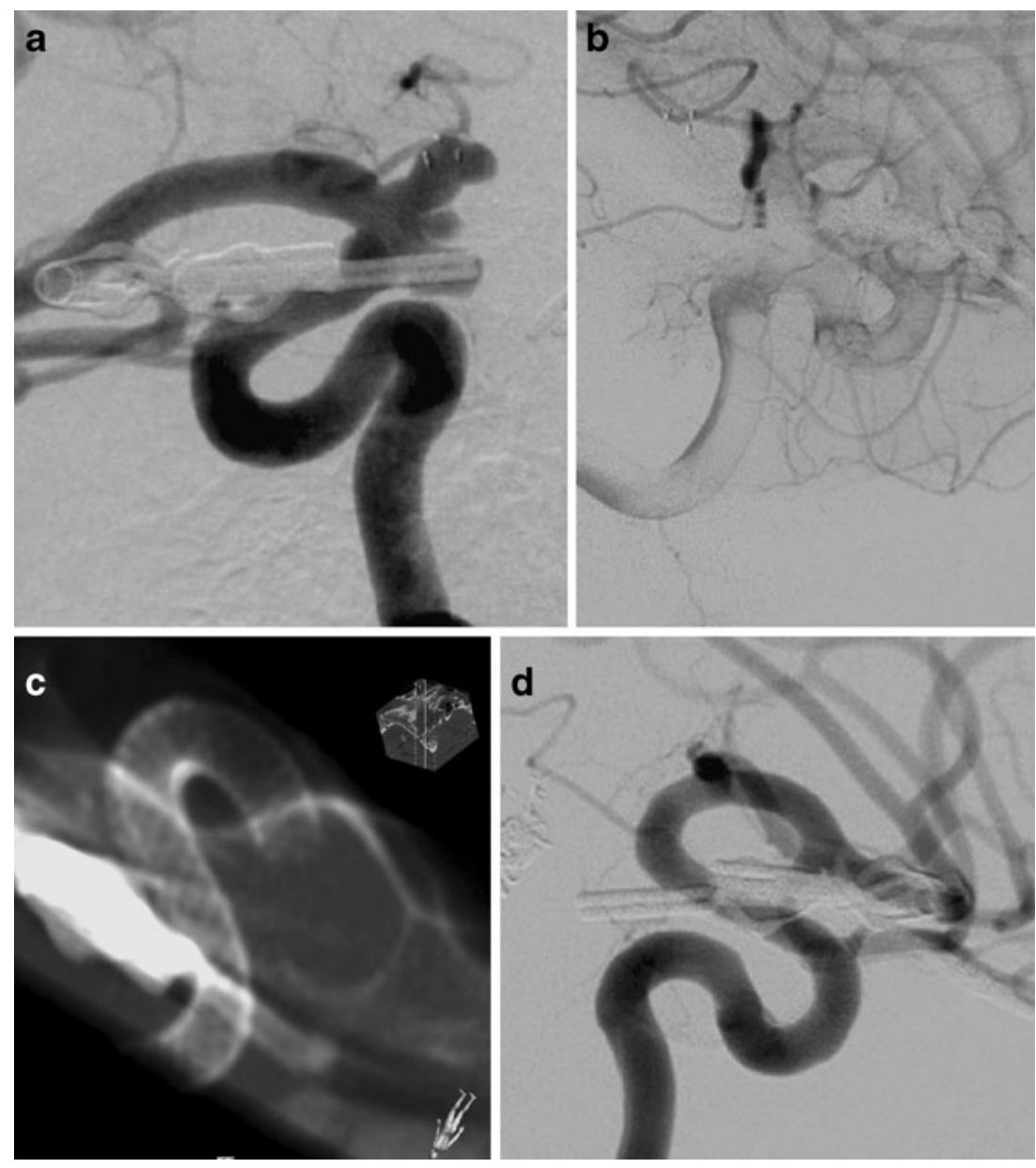

Patients 15 and 40: thrombosis of PEDs, 5 and 63 days after the treatment

Patients 18 and 34: intracerebral hemorrhages related to the dependent vasculature of the target vessel following otherwise unremarkable PED procedures

Patient 5: cerebellar hemorrhage 14 months after PED treatment of a fusiform basilar trunk aneurysm with the PED reaching into the left vertebral artery, 1 day following the coil occlusion of a right vertebral artery, which prevented the complete vessel remodeling

Patient 2 (female, 61 years old) had a large wide-necked paraclinoid aneurysm (fundus, $14 \mathrm{~mm}$; neck, $11 \mathrm{~mm}$ ) of the left ICA which had caused a visual field loss. The parent artery was reconstructed with three telescoping PEDs in one treatment session with additional loose coiling of the aneurysm via a "jailed" microcatheter. A significant contrast medium stasis was observed on the final DSA run. The patient showed no neurologic impairment directly after this treatment and was discharged 3 days later. Heparinization was stopped at discharge. One day later, she complained of severe headache and an increasing visual field loss. Computed tomography showed a massive IVH.
DSA on the same day (day 3 after the treatment) showed the previously treated aneurysm with a neck remnant while the aneurysm fundus was occluded by coils and thrombus. A small amount of contrast medium was found adjacent of the coil occluded dome of the aneurysm and was considered to be the site of rupture. The patient died 5 days later (Fig.7).

Patient 15 (male, 44 years old) initially presented with an intracerebral hemorrhage from a large, partially thrombosed, dissecting aneurysm originating from the right M1 segment. The parent artery was straightened and reinforced by coaxially deploying an Enterprise and a Solitaire stent in June 2009. The subsequent follow-up DSA showed a significant thrombosis within the aneurysm, which was, however, still partially perfused. On November 26, 2009, two PEDs $[2.5 \times 20 \mathrm{~mm}$, $2.5 \times 18 \mathrm{~mm}]$ were deployed within the two previously implanted stents. The patient received dual platelet antiaggregation as described above. On December 1, 2009, 5 days after the PED procedure, he presented with severe right-sided headache without a focal neurological deficit. Magnetic resonance imaging showed several ischemic lesions within the right middle cerebral artery supply territory and DSA confirmed the complete occlusion of the right M1 segment. 

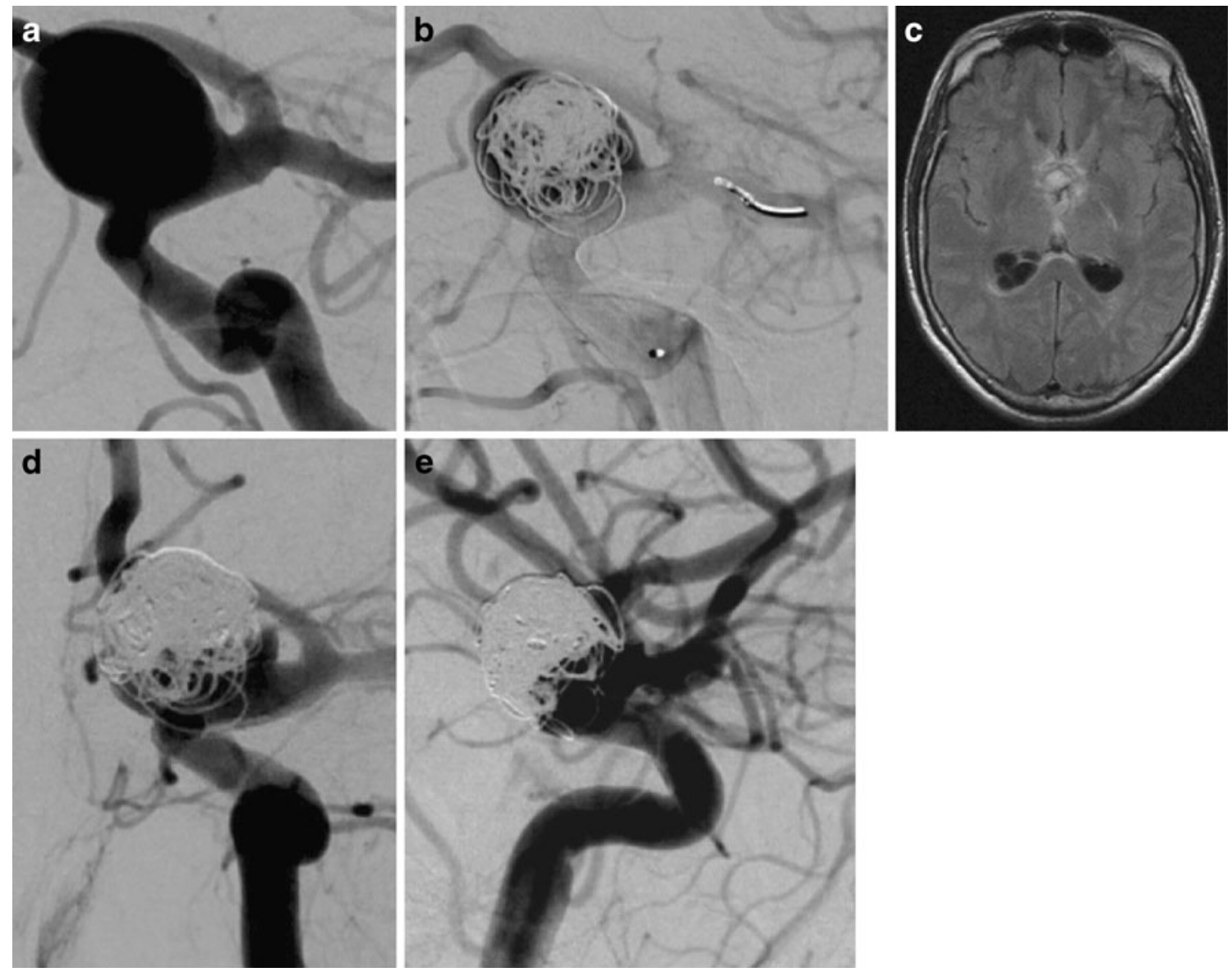

Fig. 7 Rupture of a wide-necked paraclinoid aneurysm 3 days after loose coiling and deployment of three PEDs [4/14, 4.25/20, 4.25/14]. Aneurysm prior to treatment (a) and immediately after coiling and PED implantation with stagnating contrast medium (b). Four days later, the patient presented severe headache and visual loss. MRI showed a massive intraventricular hemorrhage (c). DSA confirmed a neck remnant of the aneurysm with thrombosis of the fundus $3 \mathrm{~h}$ later (d). Late-phase DSA images revealed an accumulation of contrast medium outside the contour of the aneurysm sac adjacent to the aneurysm dome (e)
The patient recovered completely and is now free from any neurological deficit (Fig. 8).

Patient 40 (male, 71 years old) was incidentally found to carry a diffuse dilatation of the basilar artery bifurcation with a saccular component between the right superior cerebellar artery (SCA) and posterior cerebral artery (PCA) origins. Given the (SCA) irregular shape of the dilatation and in view of the partially saccular aspect, it was considered a potential source of a future bleeding. On August 13, 2009, a Solitaire AB stent was inserted into the left PCA with the proximal end of the stent in the trunk of the basilar artery. On March 24, 2010, a PED was deployed from the right PCA, crossing the Solitaire stent at the level of the basilar bifurcation and extending with the proximal end into the trunk of the basilar artery. This procedure was tolerated well and the patient was discharged in his previous condition. He was told to continue the medication of ASA and clopidogrel for 6 weeks and to continue with
ASA thereafter. Eight weeks after the treatment and 22 days after he had stopped the intake of clopidogrel, he was referred to another hospital and from there with some delay to us. At admission, the patient was comatose. DSA showed the PED and the upper basilar artery filled with thrombus. Given the time interval from clinical onset to arrival at our hospital, local intraarterial fibrinolysis was not considered a reasonable option. The attempted mechanical thrombectomy failed due to access issues, and the patient died from the sequelae of brainstem infarct 27 days later.

Patient 18 presented with recurrent attacks of severe headache. A fusiform aneurysm of the left cavernous ICA was found and treated with a combination of coils, Leo and coronary stents in April and September 2009. Angiographic follow-up in December 2009 showed a significant perfusion of the aneurysm. One PED was deployed, resulting in stagnation of contrast medium immediately thereafter. On the evening after this procedure, the patient was found 

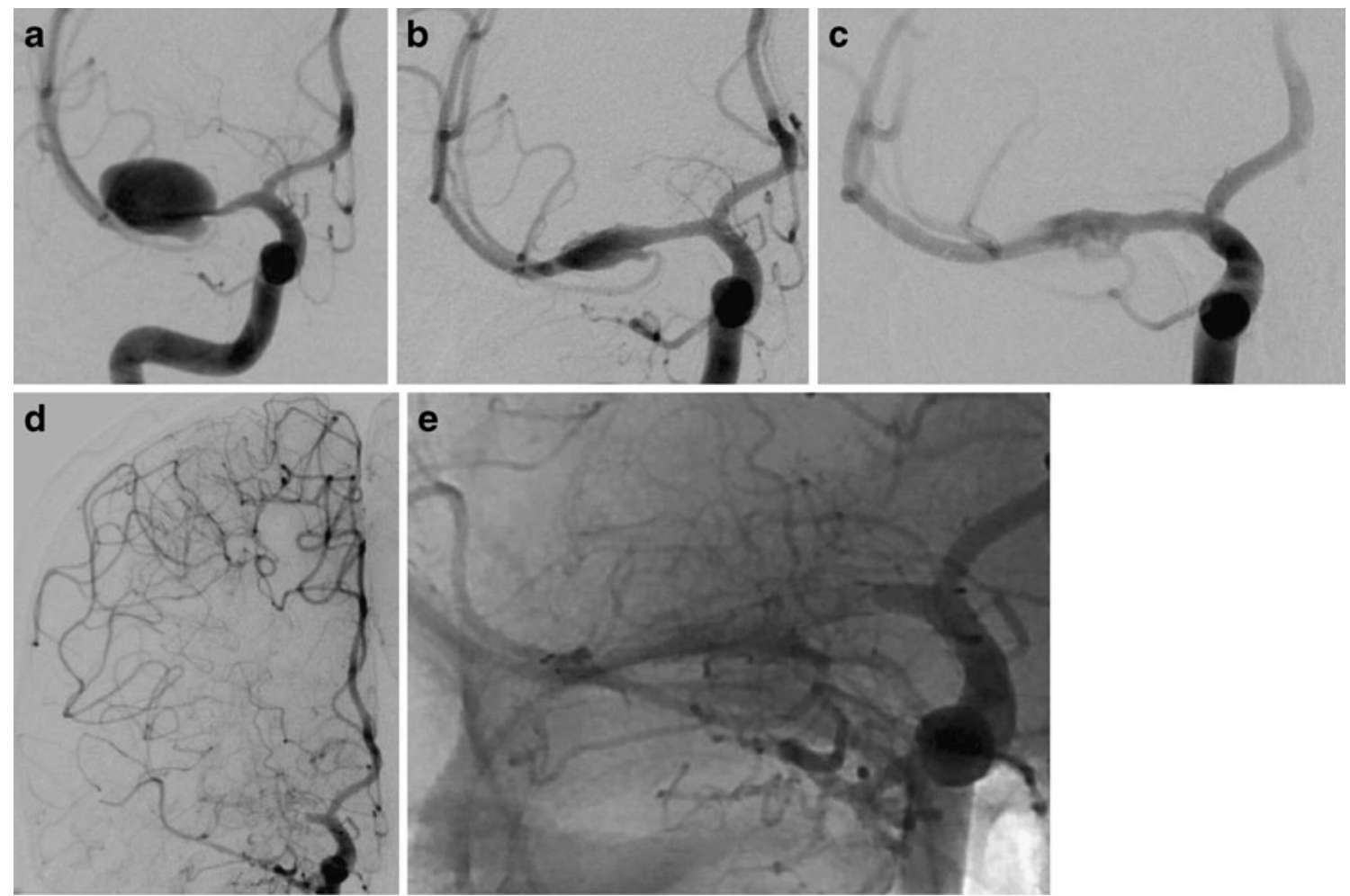

Fig. 8 Acute thrombosis of a PED deployed within two previously implanted self-expanding stents. Dissecting, partially thrombosed aneurysm of the right M1 segment (a). An Enterprise and a Solitaire stent were coaxially deployed to enforce the vessel wall and to redirect blood flow in June 2009. Follow-up DSA showed further but incomplete thrombosis of the aneurysm (b). Two PEDs $[2.5 \times 20$, $2.5 \times 18$ ] were deployed within the two stents $(\mathbf{c})$. Despite dual antiplatelet antiaggregation, acute thrombosis of PED and stents occurred 5 days later with significant MCA ischemia but without a neurological deficit $(\mathbf{d}, \mathbf{e})$ hypertensive, aphasic, and hemiplegic on her right side. CT showed a left parietal hematoma. The patient was managed conservatively and recovered to a level of complete independence with a residual expressive aphasia. Further workup showed an impaired platelet function. The dual antiaggregation was changed to $50 \mathrm{mg}$ ASA per day and $75 \mathrm{mg}$ clopidogrel every other day. With this dosage, the Multiplate test showed a sufficient platelet function inhibition. DSA in January 2011 showed a complete aneurysm occlusion. Headaches had ceased several weeks after the PED treatment.

In patient 34, a large fusiform aneurysm of the basilar trunk was treated in 2004 and in 2006 by Neuroform stent deployment and partial coiling. Angiographic follow-up showed a slow but gradual enlargement of the aneurysm. In March 2010, three PEDs were coaxially implanted. On the evening of the treatment day, the patient was hypertensive, complained of severe headache, and showed hemiataxia. CT showed a left-sided cerebellar hemorrhage, which was managed conservatively. The patients' condition returned to his previous status. Angiographic follow-up in January 2011 showed a significant reduction of the aneurysm perfusion.

For patient 5 , see the illustrative case above.

\section{Discussion}

The goal of aneurysm therapy is to isolate the vessel dilatation from cerebral circulation and restore physiological blood flow in the parent artery as far as possible. The characteristic blood flow pattern in saccular aneurysms is an inflow zone at the distal aspect of the neck and an outflow zone at the proximal side. The decline of intraaneurysmal blood circulation by flow diversion leads to deceleration of intraaneurysmal velocity and increased blood viscosity, resulting in an intraaneurysmal thrombus formation. Stents, primarily designed to assist coil occlusion of widenecked aneurysms, have shown to influence the intraaneurysmal blood flow even when used without coils [10]. The extent of intraaneurysmal flow reduction depends on the porosity and the pore density of the implanted stent(s). Conventional stents have a relatively low metal surface area coverage [11]. In contrast, the PED achieves a $30-35 \%$ metal surface area coverage [12].

The results of this series demonstrate that the PED can be a useful device for the treatment of wide-necked, fusiform, and previously incompletely treated aneurysms. The results in the 
treatment of intracranial dissections show that vessel reconstruction with the PED can be achieved instantaneously.

As with all neuroendovascular technologies, several aspects need to be considered. These include patient selection criteria, technical aspects of PED procedures, recommendations for duration and regimen of platelet antiaggregation medication and anticoagulation, complication management, prediction of aneurysm occlusion, follow-up schedule, and criteria for eventual retreatment.

\section{Patient selection}

As described above, complete aneurysm occlusion immediately after the procedure was not observed in any of the patients. Due to the delay in aneurysmal occlusion and the need for dual antiplatelet medication, PED treatment without coiling appears unsuitable for ruptured aneurysms in the acute phase. The likelihood of sufficient flow diversion in bifurcation aneurysms with incomplete coverage of the neck after placement of a PED is low. The available extrasaccular flow diverters are designed for side-wall aneurysms. The potential benefit of bidirectional flow diversion with a "kissing" implantation of two PEDs or a combination with conventional stents needs to be determined. Other techniques (e.g., microsurgical clipping or stent-assisted coil occlusion) appear preferable for the treatment of bifurcation aneurysms.

Flow diversion should currently be regarded as an endovascular treatment option for wide-necked side-wall and fusiform aneurysms or remnants of previously treated aneurysms. Since both catheterization and the need of endosaccular coil placement carries an increased risk of procedural rupture especially in very small and blister aneurysms located at curved vessels, flow diverter implantation offers a way to treat such aneurysms without wall contact, which should reduce the risk of periprocedural rupture [13]. Patients with an anticipated difficulty or failure of conventional microsurgical or endovascular techniques should particularly be considered for PED treatment.

\section{Technical aspects}

Unlike the Silk stent with a significant rate of deployment issues [14, 15], the PED is relatively easy to deploy. PED implantation should result in a combination of tight wall apposition and dense coverage of the target lesion. The selection of the appropriate diameter and length of the device is an essential factor. Implantation of an undersized device may result in poor wall apposition, with the potential risk of an endoleak-like phenomenon. Placement of a device that exceeds the diameter of the target artery will cause poor coverage of the lesion due to an incomplete compaction of the strands.

An accurate measurement of the target artery diameter proximal and distal to the lesion is essential for a proper device selection. The PED is available with a nominal diameter from 2.5 to $5 \mathrm{~mm}$ in $0.25-\mathrm{mm}$ increments. The diameter of the proximal segment of the target vessel and the diameter at the origin of the lesion determine the diameter of the PED. The distal vessel diameter is of minor importance in this regard. A smaller distal vessel diameter will, however, prevent full expansion and related foreshortening of the device and might be an argument for the selection of a shorter PED. Foreshortening of approximately $50 \%$ and possible device shift during deployment must be kept in mind when choosing the length of the device [15]. Inadvertent occlusion of normal side branches covered by a flow diverter has been reported for both Silk [16] and PED [17]. The reported risk with PED is, however, low [18]. Our series confirms this finding. Therefore, we use long devices that guarantee coverage of the entire lesion with sufficient proximal and distal overhang. If a very wide aneurysm neck of a diffusely dilated long vessel should be reconstructed, telescoping several PEDs is a feasible option $[19,20]$. It should be emphasized that, even with 16 PEDs deployed in one patient from our series, we encountered no technical issues or side branch occlusions. The PEDs were deployed in a telescoping way from PCA to VA with about four to five complete layers at every point of the entire distance.

To ensure full expansion and wall apposition of the PED, both the delivery catheter and the insertion wire must be pushed during the second phase of deployment. This technique allows compaction of the device strands. This may, however, be challenging to achieve in elongated vessels. In the case of sequential coaxial PED implantations, the microcatheter is reinserted in the previously deployed PED(s) using its pusher wire. Withdrawing this wire can be cumbersome if the capture coil on the pusher wire is not parallel to the longitudinal axis of the microcatheter.

A good wall apposition of the PED requires an equal diameter or at least a gradual diameter change of the target vessel. In the case of circumscribed stenosis, especially if the stenosis is high grade and/or eccentric, a noncompliant balloon angioplasty of the stenotic segment prior to the PED deployment is recommended. If the deployed PED shows poor wall apposition for whatever reason, an adaptation of the PED to the vessel wall with a compliant balloon may prevent endoleak and poor hemodynamic device function. Both types of balloon dilatation may add complexity and risk to the entire procedure. 
Recommendations for duration and regimen of platelet antiaggregation medication and anticoagulation, potential complications

A certain drawback in the treatment of aneurysms with flow diverters (and stents) is the need for antiplatelet medication at a point at which the aneurysm may not yet be excluded from circulation. SAH under antiplatelet therapy presents severe treatment challenges and frequently results in a dismal outcome. For bare metal porous stents, a dual antiplatelet regimen for 6 weeks followed by monotherapy is sufficient. A growing amount of literature reports early and delayed thromboembolic complications after flow diverter treatment. Based on these findings, as well as on our own experience, we extended the duration of dual antiplatelet medication to at least 1 year after treatment or longer until a complete occlusion of the aneurysm was confirmed.

As described above, we encountered one fatal SAH from a treated aneurysm and three parenchymal hemorrhages in the dependent brain territory after PED treatment. The underlying mechanism of aneurysm hemorrhage after flow diversion is a matter of controversy. The implantation of a flow diverter diminishes the intraaneurysmal blood flow. This hemodynamic effect per se should reduce the risk of aneurysmal rupture, even though the aneurysm wall is still exposed to pulsatile perfusion. Several hemodynamic studies proved the stent-induced reduction of aneurysmal inflow which leads to changes of the wall shear stress at the aneurysm dome [21,22]. These results are based on in vitro models or induced aneurysms in animals and are potentially not conferrable to any human anatomic and hemodynamic situation without restriction. An unfavorable valve mechanism with augmentation of inflow and/or suppression of outflow has been discussed ("hemodynamic theory") [23].

Another cause of rupture after flow diverter treatment was discussed by Turowski et al. [24]. The process of thrombus formation begins with the stagnation of blood flow, leading to the formation of red thrombus, progressing to a white thrombus, followed by subsequent thrombus organization. Red thrombi contain proteolytic enzymes and leucocytes. A large amount and a prolonged phase of red thrombus within the aneurysm without progression to an organized thrombus might promote an inflammatory reaction, eventually resulting in the disintegration of the aneurysm wall with subsequent rupture ("inflammatory theory"). The sequence of thrombus formation can be regulated in different directions by the use of antiplatelet, anticoagulant, and anti-inflammatory agents. In our patient 2 , the SAH occurred 1 day after the heparinization was stopped. In large saccular aneurysms, the extended administration of heparin for 6 weeks and steroids for 3 weeks is recommended and seems to reduce the risk of hemorrhage after flow diversion.
The additional use of coils in large aneurysms does neither improve nor disturb the process of aneurysm obliteration induced by flow diversion [12]. It is currently undetermined if previous partial coiling or clipping of large and giant aneurysms can prevent hemorrhage after flow diversion. Hemorrhagic complications were not encountered after PED deployment in the 34 pretreated saccular aneurysms in our series.

The presence of a previously deployed stent may reduce the hemodynamic effect of a PED [25]. Since delayed endothelialization has to be expected under these circumstances, an extended phase or lifetime-long dual platelet antiaggregation should be contemplated.

Delayed parenchymal hemorrhage is very rare after stent-assisted coil occlusion of intracranial aneurysms. We assume that microemboli (e.g., air) and microinfarcts during PED deployment may trigger parenchymal bleedings, which now occur with identical anticoagulation and antiaggregation protocols.

\section{Prediction of aneurysm occlusion}

The goal of endosaccular endovascular treatment of intracranial aneurysms is complete aneurysm occlusion. This can be accomplished by dense coil packing. Ideally, there is no visible contrast medium between the coil loops on the final angiographic run even though studies showed that the intrinsic aneurysm occlusion is not exceeding $30 \%$ at this point. The rate of hemorrhages from coiled aneurysms is low. Nevertheless, several series on the endovascular coil treatment of aneurysms confirmed a significant rate of retreatment [26]. The primary aim of flow diverter treatment of an aneurysm is to induce a process of intraaneurysmal thrombus formation with the final goal of complete aneurysm occlusion. Residual or unchanged aneurysmal filling is the typical finding immediately after placement of a flow-diverting device. The time frame of this process is probably determined by numerous factors but finally not predictable [16]. The number of treated lesions in our series is too small and the material is too inhomogeneous to allow the identification of lesional or procedural features predisposing for complete occlusion or persistent perfusion. Aneurysm size and geometry certainly play a significant role, assuming that smaller aneurysms will need a shorter period for complete thrombosis. The process of thrombosis might be extended in bifurcation aneurysms due to a clearly vectored inflow direction.

The effect of flow diverter implantation can be estimated by several angiographic signs that indicate a change of the intraaneurysmal flow. O'Kelly et al. proposed a grading scale for the angiographic assessment of intracranial aneurysms treated with flow diverters. The volume of contrast filling and the degree of contrast medium stagna- 
tion linked to the phases of the angiogram are the main elements of this grading scale. The duration of contrast stasis induced by a flow diverter might be a helpful tool to estimate the likelihood of complete intraaneurysmal thrombosis [9]. This might also help to determine the need of more than one flow diverter. In our series, more than 1 PED was used initially in 67 cases. Reasons for the deployment of multiple PEDs are a synchronous clearance of the parent artery and the aneurysm after placement of one PED. In large aneurysms with a minor delay of clearance after one PED, telescoping PED implantation was performed as well. There are, however, no definitive criteria when to stop telescoping PEDs. A single PED was used in cases with significant stasis of contrast medium up to the venous phase of the angiogram. This criterion still leaves plenty of space for error.

Our series includes 30 lesions treated previously with conventional stents. The assumption of a negative interaction between a PED and a previously implanted laser-cut porous stent arises specially with regard to the above-described complications. This combination of previously placed stents and PEDs may impair the wall apposition and may prevent the process of device endothelialization. This could have been a factor for the development of thrombotic parent vessel occlusion in the two above-mentioned patients. The origin of side branches from vessel dilatations is another factor that may prevent aneurysm thrombosis and vessel remodeling.

\section{Follow-up schedule and criteria for retreatment}

As mentioned above, we schedule the first follow-up angiographies 3 and 6 months after treatment. As expected from a series of this size, some patients are lost to follow-up despite serious efforts to obtain comprehensive follow-up data.

In the case of complete aneurysm occlusion at the second follow-up angiography and the absence of intimal hyperplasia within the PED, we advise the patient to continue with the dual antiplatelet therapy for an additional 6 months.

In patients with unchanged aneurysm perfusion compared to the postprocedural angiogram, we recommend deployment of additional $\operatorname{PED}(\mathrm{s})$. If the involution of the aneurysm is obviously disturbed by other vessels with competing flow, we evaluate and eventually perform the endovascular occlusion of these arteries [27]. The decision for retreatment was made individually based on the angiographic findings and the patient's age and clinical status. Incompletely coiled or clipped aneurysms will almost certainly not improve without further treatment, which makes the decision for retreatment in these cases relatively easy. Decision-making between retreatment and observation after flow diversion is more complex since the process of reverse remodeling after flow diverter treatment is rather unpredictable. This will hopefully change as soon as long-term follow-up data become available.

The need for further follow-up angiographies after a documented complete occlusion of an aneurysm or complete vessel reconstruction after intracranial dissections remains unclear. A mechanism of aneurysm recurrence after successful flow diversion is currently not known. Long-term data that determine the likelihood of recurrent perfusion after flow diverter treatment are required.

A potential argument for further angiographic follow-up after complete aneurysm occlusion is the high incidence of intimal hyperplasia that has been reported for the Silk flow diverter and for balloon expandable cobalt chromium stents $[15,28]$. The incidence of intimal hyperplasia with PED might be lower compared to stent-treated intracranial stenoses, since the PED is mostly implanted without balloon angioplasty thus avoiding a mechanical vessel injury. Animal experiments have shown a very low rate of intimal hyperplasia with PED [18]. Although we did not observe a single symptomatic intimal hyperplasia in this small series with a relatively short follow-up period, we propose our patients to perform follow-up angiographies in yearly intervals until further data on this issue are available.

\section{Conclusion}

Flow diversion using the PED is a technically straightforward and relatively safe treatment modality for the treatment of wide-necked saccular side-wall aneurysms, fusiform aneurysms, remnants of aneurysms after surgical or endovascular treatment, and dissected vessels in selected cases. Telescoping several PEDs and balloon adaptation of the PEDs to the vessel wall is frequently required. Vessel reconstruction after dissection is achieved within days. Aneurysm occlusion and vessel remodeling can take several months. Complications are not frequent but may include intraparenchymal bleeding into brain areas related to the target vessel, induced rupture of saccular aneurysms, and early as well as late acute in-stent thrombosis. An efficient dual antiaggregation is recommended for 1 year. Long-term data are still missing to predict the likelihood of aneurysmal occlusion after flow diverter treatment and to analyze the frequency of clinically relevant intimal hyperplasia within the PED. Flow diversion requires an individualized treatment strategy including also microsurgical and endosaccular interventional options and a careful anticoagulation-antiaggregation regimen.

Conflict of interest H.H. serves as a proctor for ev3 and receives a modest remuneration. The others authors have no conflict of interest. Open Access is sponsored by ev3. 
Open Access This article is distributed under the terms of the Creative Commons Attribution Noncommercial License which permits any noncommercial use, distribution, and reproduction in any medium, provided the original author(s) and source are credited.

\section{References}

1. Henkes H, Bose A, Felber S, Miloslavski E, Berg-Dammer E, Kühne D (2002) Endovascular coil occlusion of intracranial aneurysms assisted by a novel self-expandable nitinol microstent (Neuroform). Interv Neuroradiol 30:107-119

2. Shapiro M, Babb J, Becske T, Nelson PK (2008) Safety and efficacy of adjunctive balloon remodeling during endovascular treatment of intracranial aneurysms: a literature review. AJNR Am J Neuroradiol 29:1777-1781

3. Augsburger L, Farhat M, Reymond P, Fonck E, Kulcsar Z, Stergiopulos N, Rüfenacht DA (2009) Effect of flow diverter porosity on intraaneurysmal blood flow. Clin Neuroradiol 19:204-214

4. Henkes H, Reinartz J, Preiss H, Miloslavski E, Kirsch M, Kühne D (2006) Endovascular treatment of small intracranial aneurysms: three alternatives to coil occlusion. Minim Invasive Neurosurg 49:65-69

5. Felber S, Henkes H, Weber W, Miloslavski E, Brew S, Kühne D (2004) Treatment of extracranial and intracranial aneurysms and arteriovenous fistulae using stent grafts. Neurosurgery 55:631638

6. Pavlisa G, Ozretic D, Murselovic T, Pavlisa G, Rados M (2010) Sole stenting of large and giant intracranial aneurysms with selfexpanding intracranial stents-limits and complications. Acta Neurochir (Wien) 152:763-769

7. Leonardi M, Dall'olio M, Princiotta C, Simonetti L (2008) Treatment of carotid siphon aneurysms with a microcell stent. A case report Interv Neuroradiol 14:429-434

8. Fiorella D, Hsu D, Woo HH, Tarr RW, Nelson PK (2010) Very late thrombosis of a pipeline embolization device construct: case report. Neurosurgery 67(3 Suppl Operative):onsE313-onsE314

9. O'Kelly CJ, Krings T, Fiorella D, Marotta TR (2010) A novel grading scale for the angiographic assessment of intracranial aneurysms treated using flow diverting stents. Interv Neuroradiol 16:133-137

10. Benndorf G, Claus B, Strother CM, Chang L, Klucznik RP (2006) Increased cell opening and prolapse of struts of a neuroform stent in curved vasculature: value of angiographic computed tomography: technical case report. Neurosurgery 58(4 Suppl 2):ONS-E380

11. Krischek Ö, Miloslavski E, Fischer S, Shrivastava S, Henkes H (2011) A comparison of functional and physical properties of selfexpanding intracranial stents [Neuroform, Wingspan, Solitaire, Leo(+), Enterprise]. Minim Invasive Neurosurg 54:21-28

12. Szikora I, Berentei Z, Kulcsar Z, Marosfoi M, Vajda ZS, Lee W, Berez A, Nelson PK (2010) Treatment of intracranial aneurysms by functional reconstruction of the parent artery: the Budapest experience with the pipeline embolization device. AJNR Am J Neuroradiol 31:1139-1147

13. Kulcsár Z, Wetzel SG, Augsburger L, Gruber A, Wanke I, Rüfenacht DA (2010) Effect of flow diversion treatment on very small ruptured aneurysms. Neurosurgery 67:789-793

14. Byrne JV, Beltechi R, Yarnold JA, Birks J, Kamran M (2010) Early experience in the treatment of intra-cranial aneurysms by endovascular flow diversion: a multicentre prospective study. PLoS One 5(9). pii: e12492

15. Lubicz B, Collignon L, Raphaeli G, Pruvo JP, Bruneau M, De Witte O, Leclerc X (2010) Flow-diverter stent for the endovascular treatment of intracranial aneurysms: a prospective study in 29 patients with 34 aneurysms. Stroke 41:2247-2253

16. Kulcsár Z, Ernemann U, Wetzel SG, Bock A, Goericke S, Panagiotopoulos V, Forsting M, Ruefenacht DA, Wanke I (2010) High-profile flow diverter (Silk) implantation in the basilar artery: efficacy in the treatment of aneurysms and the role of the perforators. Stroke 41:1690-1696

17. van Rooij WJ, Sluzewski M (2010) Perforator infarction after placement of a pipeline flow-diverting stent for an unruptured A1 aneurysm. AJNR Am J Neuroradiol 31:E43-E44

18. Kallmes DF, Ding YH, Dai D, Kadirvel R, Lewis DA, Cloft HJ (2009) A second-generation, endoluminal, flow-disrupting device for treatment of saccular aneurysms. AJNR Am J Neuroradiol 30:1153-1158

19. Fiorella D, Woo HH, Albuquerque FC, Nelson PK (2008) Definitive reconstruction of circumferential, fusiform intracranial aneurysms with the pipeline embolization device. Neurosurgery 62:1115-1120

20. Fiorella D, Kelly ME, Albuquerque FC, Nelson PK (2009) Curative reconstruction of a giant midbasilar trunk aneurysm with the pipeline embolization device. Neurosurgery 64:212-217

21. Trager AL, Sadasivan C, Seong J, Lieber BB (2009) Correlation between angiographic and particle image velocimetry quantifications of flow diverters in an in vitro model of elastase-induced rabbit aneurysms. J Biomech Eng 131:034506

22. Radaelli AG, Augsburger L, Cebral JR, Ohta M, Rüfenacht DA, Balossino R, Benndorf G, Hose DR, Marzo A, Metcalfe R, Mortier P, Mut F, Reymond P, Socci L, Verhegghe B, Frangi AF (2008) Reproducibility of haemodynamical simulations in a subject-specific stented aneurysm model - a report on the Virtual Intracranial Stenting Challenge 2007. J Biomech 41:2069-2081

23. Cebral JR, Mut F, Raschi M, Scrivano E, Ceratto R, Lylyk P, Putman CM (2011) Aneurysm rupture following treatment with flow-diverting stents: computational hemodynamics analysis of treatment. AJNR Am J Neuroradiol 32:27-33

24. Turowski B, Macht S, Kulcsár Z, Hänggi D, Stummer W (2011) Early fatal hemorrhage after endovascular cerebral aneurysm treatment with a flow diverter (SILK-Stent): do we need to rethink our concepts? Neuroradiology 53:37-41

25. Lylyk P, Miranda C, Ceratto R, Ferrario A, Scrivano E, Luna HR, Berez AL, Tran Q, Nelson PK, Fiorella D (2009) Curative endovascular reconstruction of cerebral aneurysms with the pipeline embolization device: the Buenos Aires experience. Neurosurgery 64:632-642

26. Henkes H, Fischer S, Liebig T, Weber W, Reinartz J, Miloslavski E, Kühne D (2006) Repeated endovascular coil occlusion in 350 of 2759 intracranial aneurysms: safety and effectiveness aspects. Neurosurgery 58:224-232

27. Liebig T, Henkes H (2008) Re- and deconstruction: staged treatment of large fusiform aneurysms of the vertebrobasilar junction by stent deployment and subsequent coil occlusion of one vertebral artery. Clin Neuroradiol 18:255-260

28. Vajda Z, Miloslavski E, Güthe T, Schmid E, Schul C, Albes G, Henkes H (2010) Treatment of intracranial atherosclerotic arterial stenoses with a balloon-expandable cobalt chromium stent (Coroflex Blue): procedural safety, efficacy, and midterm patency. Neuroradiology 52:645-651 